\title{
Time analysis for temporary gravitational capture
}

\section{Stable orbits}

\author{
O. C. Winter and E. Vieira Neto* \\ 1 Grupo de Dinâmica Orbital \& Planetologia, UNESP, CP 205, Guaratinguetá, CEP 12500-000, SP, Brazil \\ 2 e-mail: ernesto@feg.unesp.br
}

Received 20 April 2001 / Accepted 20 August 2001

\begin{abstract}
In a previous work, Vieira Neto \& Winter (2001) numerically explored the capture times of particles as temporary satellites of Uranus. The study was made in the framework of the spatial, circular, restricted threebody problem. Regions of the initial condition space whose trajectories are apparently stable were determined. The criterion adopted was that the trajectories do not escape from the planet during an integration of $10^{5}$ years. These regions occur for a wide range of orbital initial inclinations $(i)$. In the present work it is studied the reason for the existence of such stable regions. The stability of the planar retrograde trajectories is due to a family of simple periodic orbits and the associated quasi-periodic orbits that oscillate around them. These planar stable orbits had already been studied (Hénon 1970; Huang \& Innanen 1983). Their results are reviewed using Poincaré surface of sections. The stable non-planar retrograde trajectories, $110^{\circ} \leq i<180^{\circ}$, are found to be tridimensional quasi-periodic orbits around the same family of periodic orbits found for the planar case $\left(i=180^{\circ}\right)$. It was not found any periodic orbit out of the plane associated to such quasi-periodic orbits. The largest region of stable prograde trajectories occurs at $i=60^{\circ}$. Trajectories in such region are found to behave as quasi-periodic orbits evolving similarly to the stable retrograde trajectories that occurs at $i=120^{\circ}$.
\end{abstract}

Key words. planets and satellites: general - astrometry - celestial mechanics

\section{Introduction}

In a previous paper (Vieira Neto \& Winter 2001) we have studied the problem of temporary gravitational capture of Uranus hypothetical satellites. The approach adopted was to compute the capture times for a significant part of the initial conditions space. The results were presented in terms of gray-coded diagrams of semi-major axis $(a)$ versus eccentricity $(e)$ taking fixed values for the other initial orbital elements. One of the important features presented in the results were the regions of prisoner trajectories, trajectories that did not escape for an integration period of $10^{5}$ years.

The main purpose of the present work is try to better understand the reason for the existence of such regions of apparent stability. In this paper we concentrate our efforts on the apparently stable regions that appeared for initial conditions of retrograde orbits at pericenter in opposition and for other values of initial inclination (Vieira Neto \& Winter 2001).

The case of planar retrograde periodic orbits $(i=$ $180^{\circ}$ ) has been studied by several authors (Hénon 1965a;

\footnotetext{
Send offprint requests to: O. C. Winter, e-mail: ocwinter@feg. unesp.br

* UNESP Post Doctor Program.
}

1965b, 1966a, 1966b, 1969, 1970; Broucke 1968; Jefferys 1971; Benest 1971, 1974, 1975, 1976, 1977). In Sect. 2 we revisit this problem using Poincaré surface of section to locate and measure the size of the stable regions and compare them to the results derived from capture times (Vieira Neto \& Winter 2001). Then, we explore the apparently stable regions in the case of tridimensional retrograde orbits in Sect. 3.1. The case of tridimensional prograde orbits is analyzed in Sect. 3.2.

In the present work, we do not consider two kinds of stationary large prograde orbits, the $g_{1}, g_{2}$ family (Hénon 1970), also called S and N (Gorkavyi 1993), since they are inside the lobe defined by the Lagragean point $L_{1}$.

\section{Planar retrograde orbits}

The region of the initial conditions space with apparently stable retrograde orbits determined for the Sun-Jupiter mass ratio (Huang \& Innanen 1983; Brunini 1996) resembles the results for the Sun-Uranus mass ratio (Vieira Neto \& Winter 2001). In Fig. 1 we reproduce a diagram indicating the initial conditions in terms of $a$ versus $e$, taking $i=\omega=\Omega=0^{\circ}$ and $\tau=0$ ( $i$ is the orbital inclination, $\omega$ is the argument of pericenter, $\Omega$ is the longitude 
of the ascending node and $\tau$ is the time of pericenter's passage), with capture times longer than $10^{5}$ years (gray area), called prisoner trajectories. This result is reproduced from Vieira Neto \& Winter (2001). The black lines correspond to contours of fixed values of Jacobi constant. The points $\mathrm{A}, \mathrm{B}$ and $\mathrm{C}$ correspond to three particular periodic orbits to be discussed later on this paper.

In order to better understand the reason for the existence of such apparently stable region we used the technique of Poincaré surfaces of section. Following is presented the method and the results.

\subsection{Poincaré surfaces of section}

In order to determine the orbital elements of a particle at any instant it is necessary to know its position $(x, y)$ and velocity $(\dot{x}, \dot{y})$; these correspond to a point in a four dimensional phase space. The existence of the Jacobi constant implies the existence of a three dimensional surface in the four dimensional space. For a fixed value of the Jacobi constant only three of the four quantities are needed, say $x, y$ and $\dot{x}$, since the other one, $\dot{y}$ is determined, up to a change in sign, by the Jacobi constant. By defining a plane, say $y=0$, in the resulting three dimensional space, the values of $x$ and $\dot{x}$ can be plotted every time the particle has $y=0$. The ambiguity in the sign of $\dot{y}$ is removed by considering only those crossing with a fixed sign of $\dot{y}$. The section is obtained by fixing a plane in phase space and plotting the points when the trajectory intersects this plane in a particular direction. This technique is good at determining the regular or chaotic nature of the trajectory. In the Poincaré map, quasi-periodic orbits appear as closed well-defined curves. Periodic orbits appear as isolated single points inside such islands. Any "fuzzy" distribution of points in the surface of section implies that the trajectory is chaotic.

This is the method of the Poincaré surface of section or the Poincaré map. It has been largely used to determine the location and size of regular and chaotic regions in the phase space of the circular restricted three-body problem. Hénon (1965ab, 1966a,b) explored the problem of primaries with equal masses $(\mu=0.5)$ and later (Hénon $1969,1970)$ he considered the Hill's case. More recently, the interior (Winter \& Murray 1994a, 1997a) and exterior (Winter \& Murray 1994b, 1997b) regions of the SunJupiter case $(\mu=0.001)$ were fully explored using this same technique.

In order to explore the region of apparent stability shown in Fig. 1 we produced about thirty Poincaré surfaces of section. The range of $C_{\mathrm{j}}$ considered coincide with the range needed to cover the gray area given in Fig. 1 $\left(2.9991 \leq C_{\mathrm{j}} \leq 3.0050\right)$. A sample of the Poincaré surfaces of section generated are given in Fig. 2 . The center of the islands for each surface of section corresponds to one periodic orbit. They are members of a family of simple retrograde periodic orbits called family "f" (Stromgren 1935).

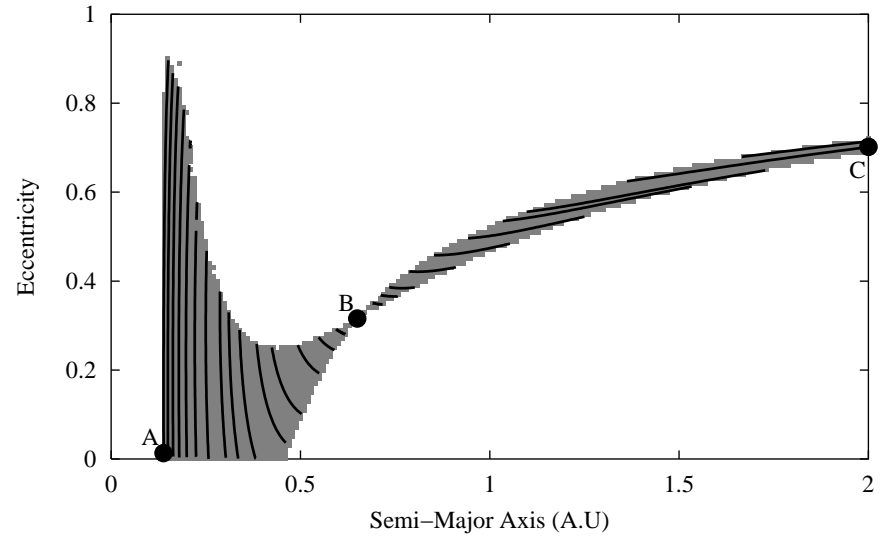

Fig. 1. Diagram indicating the initial conditions in terms of $a$ versus $e$, taking $i=\omega=\Omega=0^{\circ}$ and $\tau=0$, with capture times longer than $10^{5}$ years (gray area). This plot was reproduced using the results from Vieira Neto \& Winter (2001). The black lines correspond to contours of fixed values of Jacobi constant. The points $\mathrm{A}, \mathrm{B}$ and $\mathrm{C}$ correspond to three particular periodic orbits.

\subsection{Periodic orbits and stable regions}

The center of the small island shown in the surface of section with $C_{\mathrm{j}}=2.9999$ corresponds to the periodic orbit indicated by point B in Fig. 1. The largest islands curves give the size of the regular regions. The evolution of the size of the islands as the Jacobi constant decreases shows that the size of the regular regions decrease up to $C_{\mathrm{j}}=$ 2.9999 and then increase again (Fig. 2). The phenomenon which occurs at $C_{\mathrm{j}}=2.9999$ has already been verified by other authors (Hénon 1970; Winter 2000) and it is due to the appearance of a 3:1 resonance (Arnold 1987). Note the change in the shape of the islands before and after $C_{\mathrm{j}}=$ 2.9999. That is equivalent to the change of the contours before and after point B (Fig. 1), from almost vertical lines to almost horizontal lines.

The size and location of the stable region associated to pericentric initial conditions can be determined by measuring the size and location of the largest islands of the surface of section. The horizontal width of the largest island for each surface of section (Fig. 2) can be converted into a pair of values of semi-major axis and eccentricity (Winter \& Murray 1997a; Winter 2000). Proceeding with such computations, our results reveal that the stable regions from the surfaces of section corresponds exactly to the length of its $C_{\mathrm{j}}$ contour given in Fig. 1. Therefore, the apparently stable regions obtained computing capture times (Vieira Neto \& Winter 2001) are in fact stable regions due to the quasi-periodic orbits associated to the periodic orbits of family "f".

A sample of trajectories of this family of periodic orbits is given in Fig. 3. The trajectories chosen are the three simple periodic orbits A, B and C indicated in Fig. 1. From the trajectories draw in the baricentric rotating system we can see that they originated from periodic orbits of the first kind ( $e \approx 0$, Hénon 1966a). The initial eccentricity of orbit $\mathrm{C}$ is higher than that of orbit $\mathrm{B}$, which is higher 

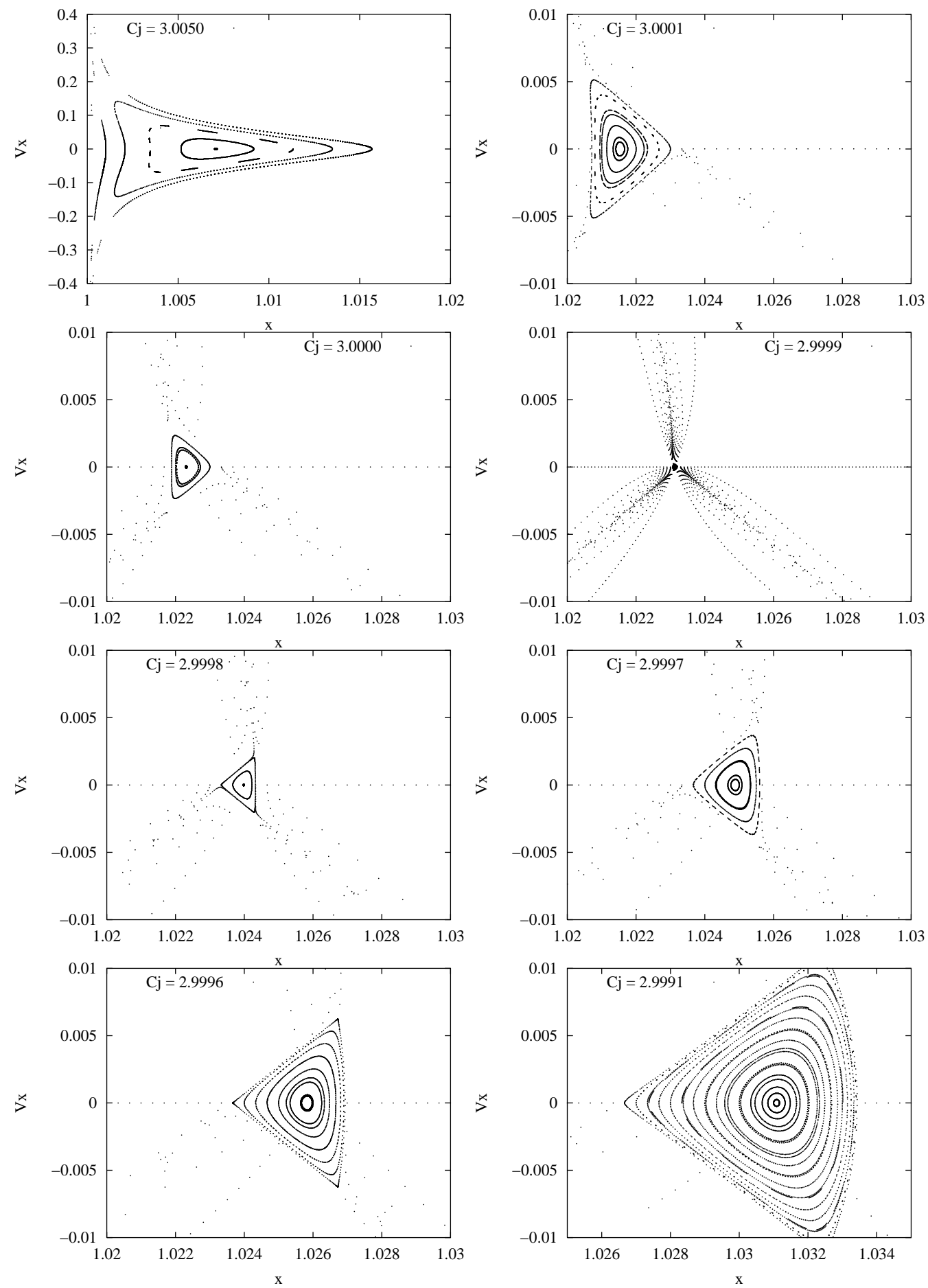

Fig. 2. Set of Poincaré surfaces of section for different values of Jacobi constant, $C_{\mathrm{j}}$. The range of $C_{\mathrm{j}}$ considered in these plots coincide with the range needed to cover the gray area given in Fig. 1. The horizontal width of the largest island for each surface of section corresponds to the length of its $C_{\mathrm{j}}$ contour given in Fig. 1 . The center of the small island shown in the surface of section with $C_{\mathrm{j}}=2.9999$ corresponds to the periodic orbit indicated by point B in Fig. 1 . Note the change in the shape of the islands before and after $C_{\mathrm{j}}=2.9999$. That is equivalent to the change of the contours before and after point B (Fig. 1), from almost vertical lines to almost horizontal lines. Also note the difference on the scales of $a$ for the first (top left corner) and the last (bottom right corner) plots.

than that of orbit A. However, the trajectories show an oval shape centered in the planet. The usual effects of such eccentricities can only be clearly seen from the trajectories draw in the non-rotating planetocentric system.
The differences on the quasi-periodic orbits before and after point $\mathrm{B}$ can be better visualized comparing the regular trajectories of two extreme cases. In Figs. 4 and 5 we present trajectories in the baricentric rotating system for 

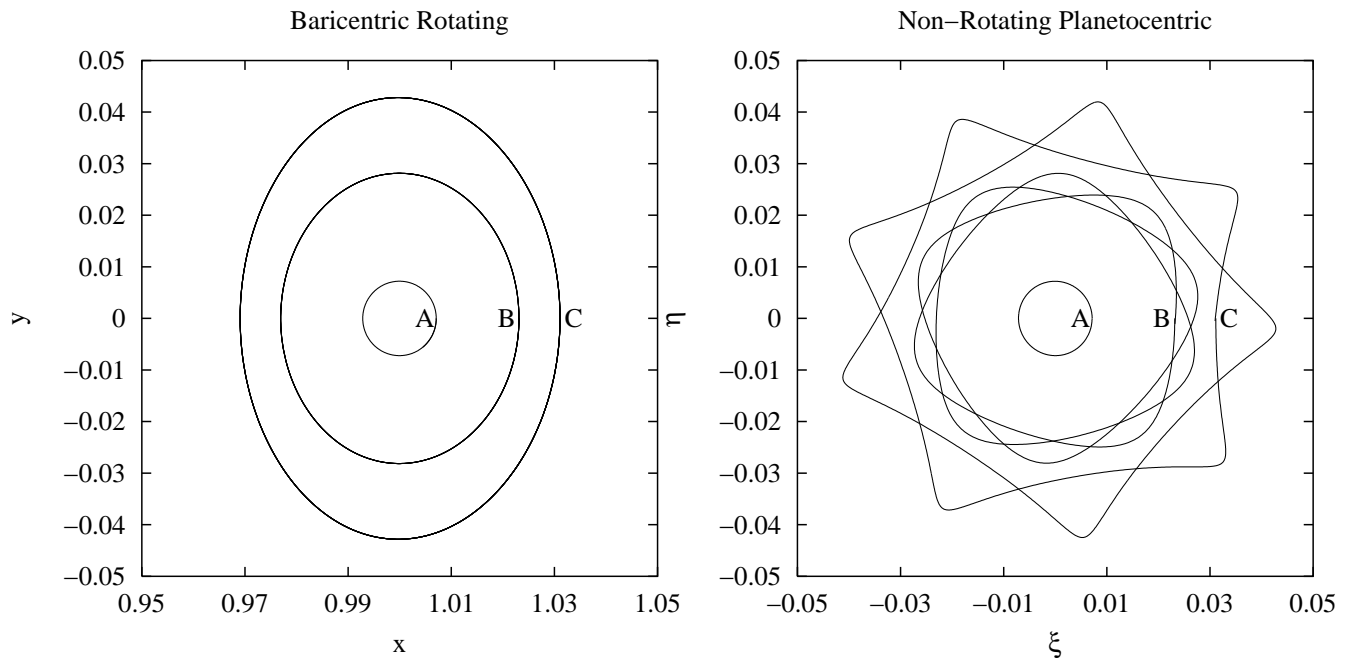

Fig. 3. Trajectories of the three simple periodic orbits A, B and C indicated in Fig. 1. From the trajectories draw in the baricentric rotating system we can see that they originated from periodic orbits of the first kind $(e \approx 0)$. The initial eccentricity of orbit $\mathrm{C}$ is higher than that of orbit $\mathrm{B}$, which is higher than that of orbit A. However, the trajectories show an oval shape centered in the planet. The usual effects of such eccentricities can only be clearly seen from the trajectories in the non-rotating planetocentric system.
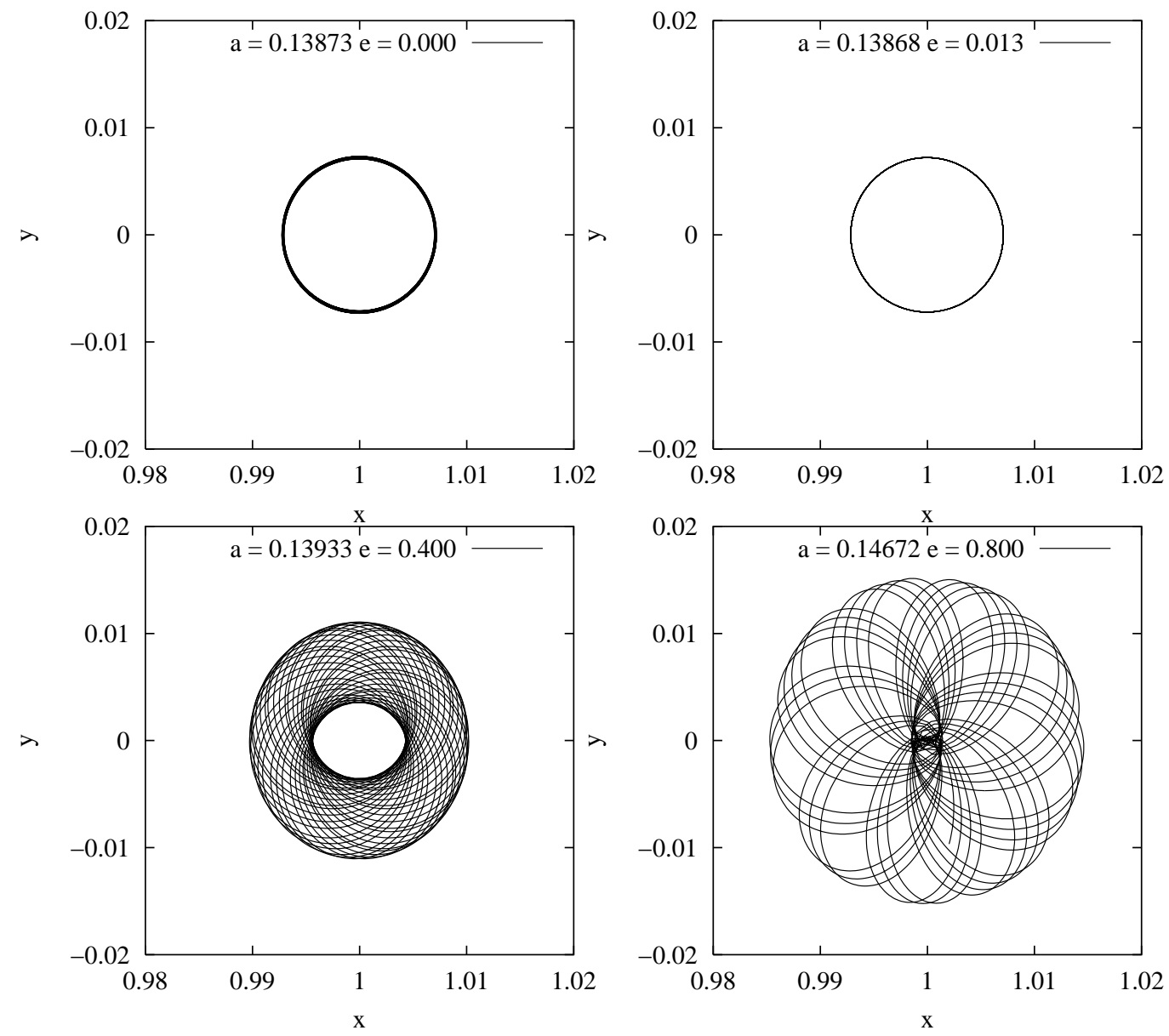

Fig. 4. Sample of stable orbits with $C_{\mathrm{j}}=3.005$. All these trajectories have initial $a \approx 0.14$ AU and their initial eccentricity is indicated in each plot. The trajectory corresponding to point A in Fig. 1 is the periodic orbit with initial $e=0.013$. The other three trajectories shown are quasi-periodic orbits oscillating around the periodic orbit. Note that the amplitude of oscillation increases as the initial eccentricity gets far from the value of the periodic orbit's eccentricity. 

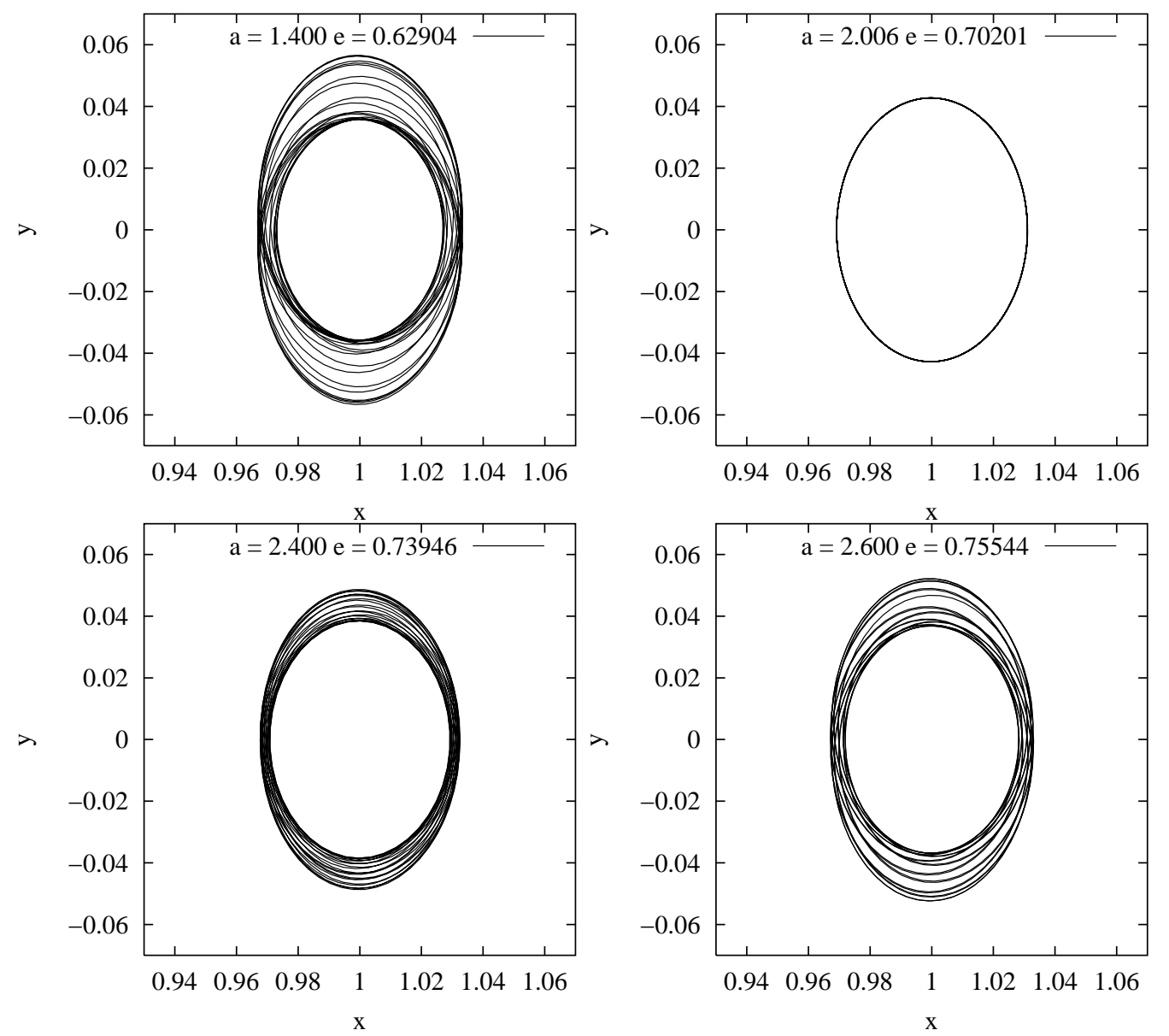

Fig. 5. Sample of stable orbits with $C_{\mathrm{j}}=2.9991$. All these trajectories have initial eccentricity between 0.6 and 0.8 , while their initial semi-major axis is indicated in each plot. The trajectory corresponding to point $\mathrm{C}$ in Fig. 1 is the periodic orbit with $a=2.006 \mathrm{AU}$. The other five trajectories shown are quasi-periodic orbits oscillating around the periodic orbit. Note that the amplitude of oscillation increases as the initial semi-major axis gets far from the value of the periodic orbit's semi-major axis.

the cases with $C_{\mathrm{j}}=3.0050$ and $C_{\mathrm{j}}=2.9991$. A sample of stable orbits with $C_{\mathrm{j}}=3.005$ is presented in Fig. 4. All these trajectories have initial $a \approx 0.1387 \mathrm{AU}$ and their initial eccentricity is indicated in each plot. The trajectory corresponding to point A in Fig. 1 is the periodic orbit with initial $e=0.013$. The other five trajectories shown are quasi-periodic orbits oscillating around the periodic orbit. Note that the amplitude of oscillation increases as the initial eccentricity gets far from the value of the periodic orbit's eccentricity.

A sample of stable orbits with $C_{\mathrm{j}}=2.9991$ is presented in Fig. 5. All these trajectories have high initial eccentricity, between 0.6 and 0.8 , while their initial semi-major axis are indicated in each plot. The trajectory corresponding to point $\mathrm{C}$ in Fig. 1 is the periodic orbit with $a=2.006 \mathrm{AU}$. The other five trajectories shown are quasi-periodic orbits oscillating around the periodic orbit. Note that the amplitude of oscillation increases as the initial semi-major axis gets far from the value of the periodic orbit's semi-major axis.

The limiting case of this kind of periodic orbit can be seen as epicyclic orbits, as described in Chauvineau \& Mignard (1990).

\section{Stability of regions out of the plane}

Once it is understood the reason for the stability of the planar prisoner trajectories found in Vieira Neto \& Winter (2001) we move on to the case of prisoner trajectories out of the plane. In the work of Vieira Neto \& Winter (2001) are presented diagrams of capture times in terms of initial $a$ versus $e$, taking $\omega=\Omega=0^{\circ}, \tau=0$. The initial inclination, $i$, for each diagram was fixed in the range $10^{\circ} \leq i \leq 180^{\circ}$, taking $\Delta i=10^{\circ}$ (Fig. 3 of Vieira Neto \& Winter 2001). Since we are interested only on the stability of the prisoner trajectories (capture times longer than $10^{5}$ years). Therefore, we have reproduced new diagrams showing just the regions with prisoner trajectories (Fig. 6). The adopted initial inclination is indicated in each plot. Obviously, the diagrams do not include the cases without significant regions of prisoner trajectories $\left(i=10^{\circ}, 90^{\circ}\right.$ and $\left.100^{\circ}\right)$. These plots were reproduced using the results from Vieira Neto \& Winter (2001). Note that the plots of the last row covers a wider range of semimajor axes, and the last plot shows the same region presented in Fig. 1. 

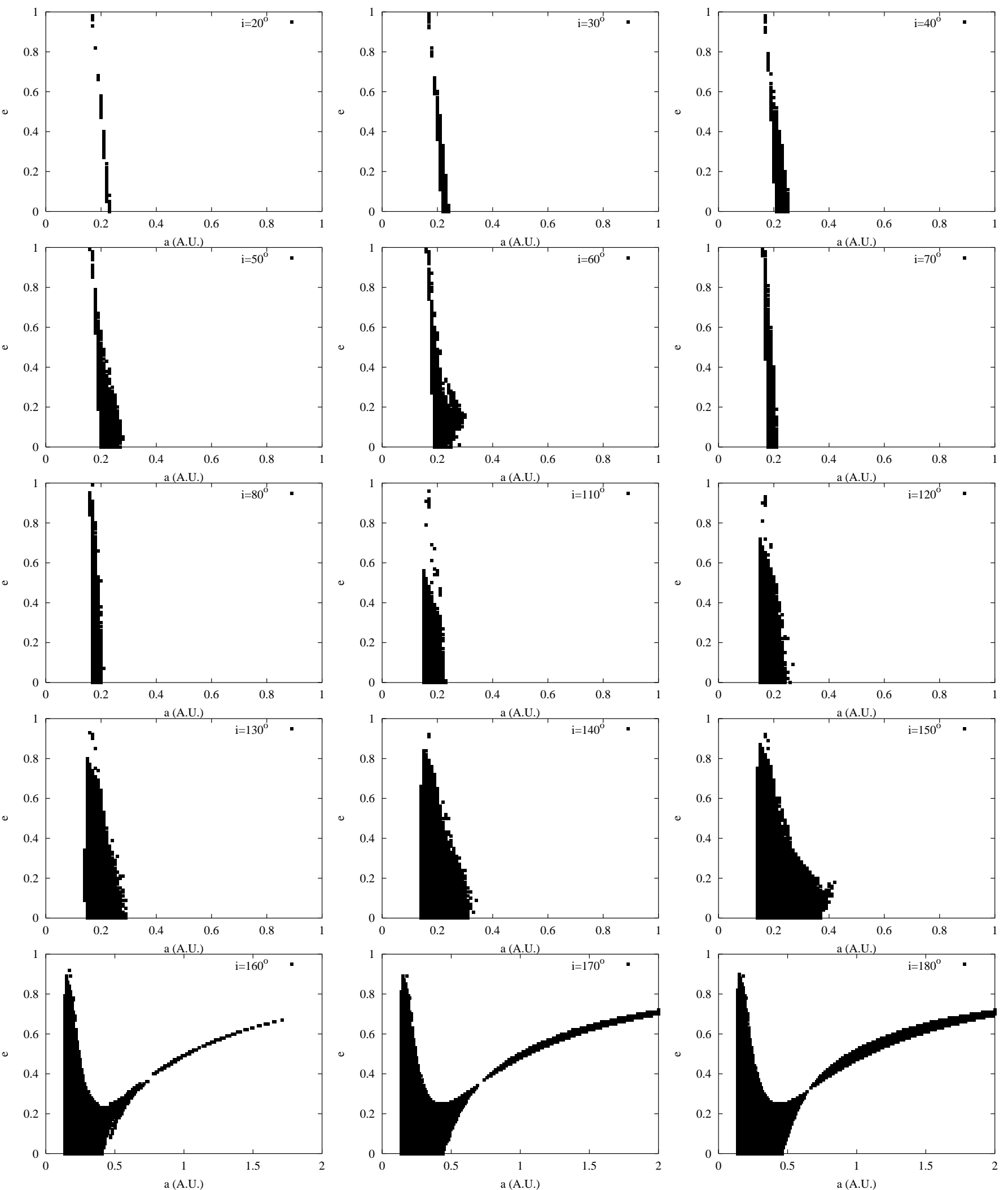

Fig. 6. Set of diagrams showing the initial conditions in terms of $a$ versus $e$, taking $\omega=\Omega=0^{\circ}$ and $\tau=0$, with capture times longer than $10^{5}$ years (black areas). Each plot has the adopted initial inclination indicated. These plots were reproduced using the results from Vieira Neto \& Winter (2001). Note that the plots of the last row covers a wider range of semi-major axes. 

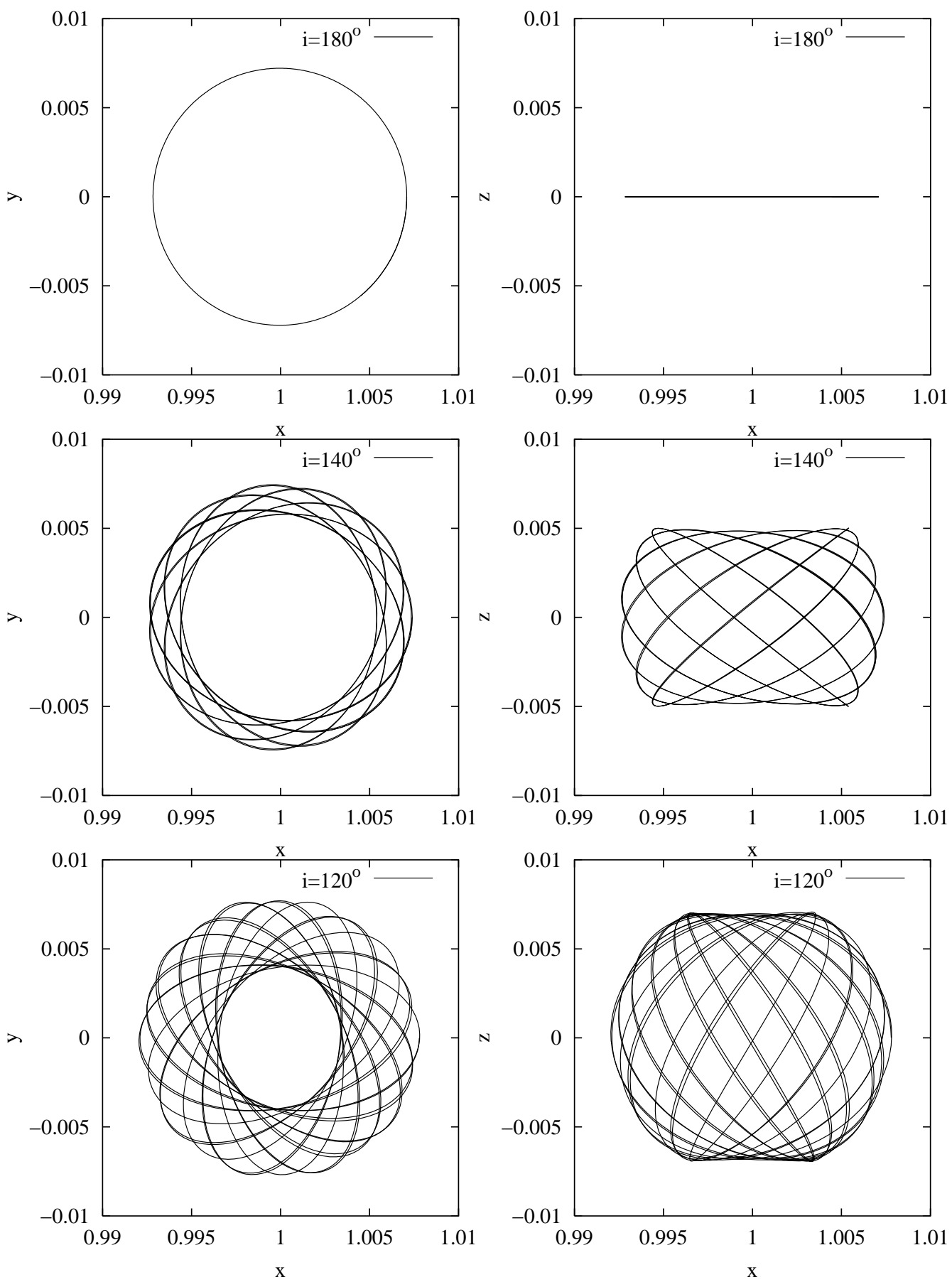

Fig. 7. Sample of stable orbits with the same initial eccentricity, $e=0.013$. The initial inclination of each trajectory is indicated on the plot. The trajectories are shown projected in the $X Y$ plane (left column) and in the $X Z$ plane (right column). The periodic orbit $\mathrm{A}$ is shown in the first pair of plots. The other two pairs of plots present 3-D quasi-periodic orbits around the planar periodic one. Note that the amplitude of oscillation increases as the initial inclination decreases.

From Fig. 6 we can see that the largest region for prograde orbits occurs at $i=60^{\circ}$. While in the case of retrograde orbits the largest region occurs at $i=$ $180 \mathrm{deg}$. We can also note that there is no prograde orbit with $a \geq 0.32 \mathrm{AU}$, while there are retrograde orbits with $a \geq 2$ AU. Hamilton \& Burns (1991) studied the forces components acting on the problem of initially circular trajectories. They concluded that in the planar case retrograde orbits should be stable out to greater distances than prograde ones since in the former situation the Coriolis acceleration is inward while in the latter it is outward. Hamilton \& Krivov (1997) derived an analytical approach in order to explain the preference for stability of the planar retrograde orbits rather than the prograde ones. The results of the previous section demonstrated that the reason for the existence of the region of the planar 

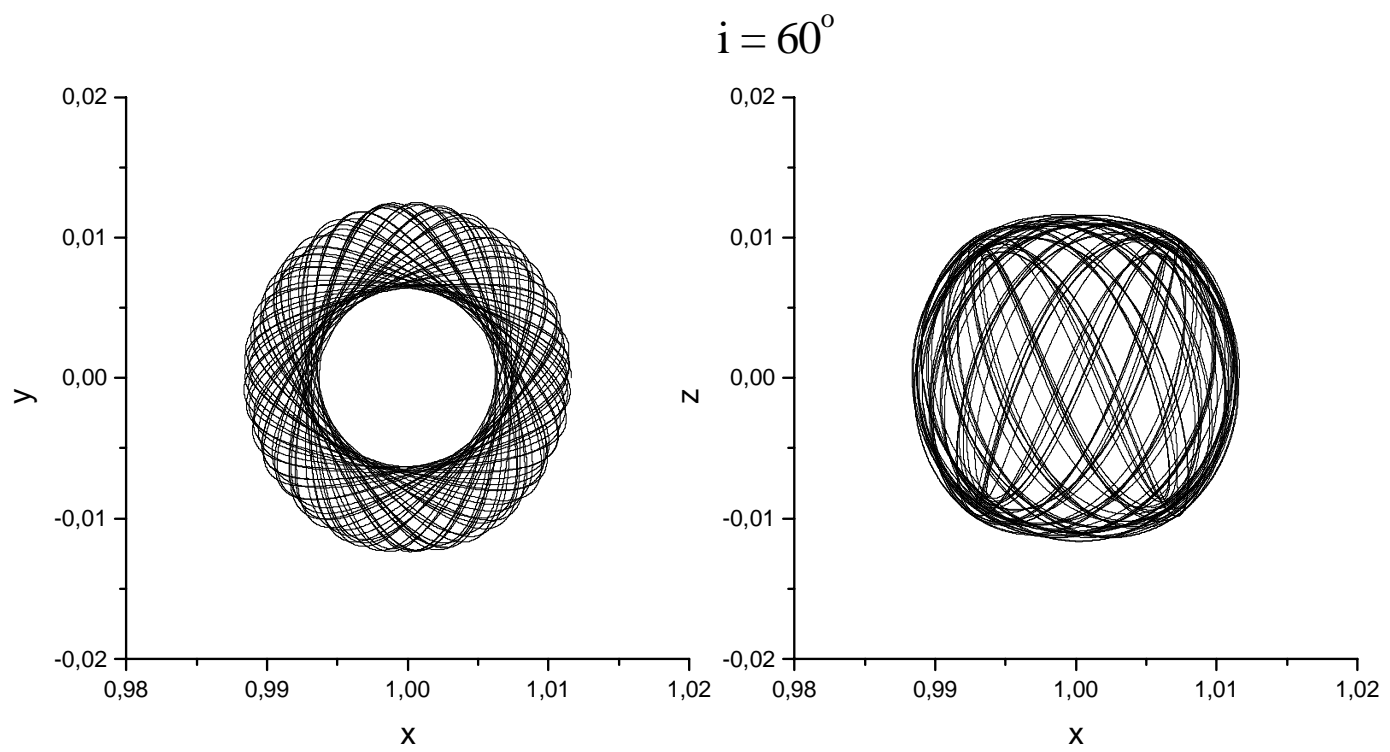

Fig. 8. A regular orbit with initial inclination of $60^{\circ}$. The trajectory is shown projected in the $X Y$ plane (top view) and in the $X Z$ plane (edge on). Note that the behavior of this trajectory is very much similar to the quasi-periodic orbit with initial inclination of $120^{\circ}$ shown in Fig. 7, but with a larger amplitude of oscillation.

retrograde prisoner trajectories was found to be the existence of the family of periodic orbits "f" and the quasiperiodic orbits oscillating around them. Therefore, one could expect that there would be periodic orbits out of the plane in order to explain the existence of the inclined prisoner trajectories shown in Fig. 6. Therefore, we performed a search for such periodic orbits without success.

\subsection{Tridimensional retrograde orbits}

Exploring the orbital evolution of inclined retrograde prisoner trajectories we identified a pattern related to the planar periodic orbits of family "f". A sample of such orbits with the same initial eccentricity, $e=0.013$, but different initial inclinations is given in Fig. 7. The initial inclination of each trajectory is indicated on the plot. The trajectories are shown projected in the $X Y$ plane (left column) and in the $X Z$ plane (right column). The periodic orbit A is shown in the first pair of plots. The other three pairs of plots shown suggest that they are tridimensional quasiperiodic orbits oscillating around the planar periodic one. Note that the amplitude of oscillation increases as the initial inclination decreases. However, that is not the case for all the retrograde prisoner trajectories given in Fig. 6. The trajectories on the borders of the regions (black areas) do not show quasi-periodic orbit's behavior despite of the fact that these trajectories did not escape for an integration period of $10^{5}$ years. Such trajectories will escape later. The trajectories are similar to the case shown in Figs. 12 and 13 of Hamilton \& Burns (1991). That might be explained because of the problem's fractal-like nature (Murison 1989). They show a sort of sticking phenomenon (Winter \& Murray 1995).

\subsection{Tridimensional prograde orbits}

In the case of inclined prograde prisoner trajectories, the results are not much different from the inclined retrograde case. In Fig. 8 we present a regular orbit with initial inclination of $60^{\circ}$. The trajectory is shown projected in the $X Y$ plane and in the $X Z$ plane. Note that the behavior of this trajectory is very much similar to the quasi-periodic orbit with initial inclination of $120^{\circ}$ shown in Fig. 7, but with a larger amplitude of oscillation. Therefore, that would mean that the inclined prograde prisoner trajectories would be tridimensional quasi-periodic orbits oscillating around the planar periodic orbit of family " $\mathrm{f}$ ". Similarly to the inclined retrograde prisoner trajectories region, the trajectories on the borders of the regions (gray areas) do not show quasi-periodic orbit's behavior for the same reason.

Acknowledgements. We would like to thanks T. Stuchi and A. A. Corrêa for some fruitful discussions on periodic orbits out of the plane. This work was partially funded by Fapesp (Proc. 98/15025-7) and Fundunesp (Proc. 458/2000-DFP). These supports are gratefully acknowledged. We also would like to thanks Dr. N. Gorkavyi for the advice and the comments on this paper.

\section{References}

Arnold, V. I. 1987, Métodos Matemáticos da Mecânica Clássica (Editora Mir, Moscow)

Benest, D. 1971, A\&A, 13, 157

Benest, D. 1974, A\&A, 32, 39

Benest, D. 1975, A\&A, 45, 353

Benest, D. 1976, A\&A, 53, 231

Benest, D. 1977, A\&A, 54, 568

Broucke, R. 1968, JPL Technical Report, 321168

Brunini, A. 1996, Cel. Mec. Dyn. Astron., 64, 79 
Chauvineau, B., \& Mignard, F. 1990, Icarus, 83, 360

Gorkavyi, N. 1993, Astron. Lett., 19(6), 448

Hamilton, D. P., \& Burns, J. A. 1991, Icarus, 92, 118

Hamilton, D. P., \& Krivov, A. V. 1997, Icarus, 128, 241

Hénon, M. 1965a, Ann. Astr., 28, 499

Hénon, M. 1965b, Ann. Astr., 28, 992

Hénon, M. 1966a, Bull. astr., Paris, 1, fasc. 1, 57

Hénon, M. 1966b, Bull. astr., Paris, 1, fasc. 2, 49

Hénon, M. 1969, A\&A, 1, 223

Hénon, M. 1970, A\&A, 9, 24

Huang, T. Y., \& Innanen, K. A. 1983, AJ, 88, 10

Jefferys, W. H. 1971, An Atlas of Surfaces of Section for the Restricted Three Bodies University of Texas, Austin

Murison, M. A. 1989, AJ, 98, 2346

Stromgren, E. 1935, Bull. Astr., Paris, 9, 87
Vieira Neto, E., \& Winter, O. C. 2001, AJ, 122, 1, 440

Winter, O. C., \& Murray, C. D. 1994a, Atlas of the planar, circular, restricted three-body problem. I. Internal Orbits, QMW Maths Notes, No. 16 (Queen Mary and Westfield College, London, UK)

Winter, O. C., \& Murray, C. D. 1994b, Atlas of the planar, circular, restricted three-body problem. II. External Orbits, QMW Maths Notes, No. 17 (Queen Mary and Westfield College, London, UK)

Winter, O. C., \& Murray, C. D. 1995, In From Newton to Chaos, NATO ASI Series B: Phys., 336, 193

Winter, O. C., \& Murray, C. D. 1997a, A\&A, 319, 290

Winter, O. C., \& Murray, C. D. 1997b, A\&A, 328, 304

Winter, O. C. 2000, Planet. Space Sci., 48, 23 